\title{
Calcium Uptake by Intestinal Brush Border Membrane Vesicles Comparison with In Vivo Calcium Transport
}

\author{
H. P. Schedl and H. D. Wilson \\ With technical assistance of T. Miller and B. Ogesen \\ Veterans Administration Medical Center and Gastroenterology-Hepatology Laboratories, University of Iowa College of Medicine, \\ Iowa City, Iowa 52242
}

\begin{abstract}
In prior studies, we examined kinetics of steady state in vivo transepithelial calcium transport in rat and hamster. The present studies related calcium uptake by the brush border to in vivo transport. We measured calcium uptake by brush border membrane vesicles from the two species. In the rat, our prior in vivo studies had shown that (a) calcium transport was mediated, (b) no nonmediated component was detectable, and (c) $V_{\max }$ was 2.5 times greater in proximal than distal small intestine. In brush border membrane vesicles from the rat, $V_{\max }$ for the saturable component of calcium uptake was again 2.5 times greater in proximal than distal intestine. Contrasting with in vivo studies, a major nonsaturable component was present in vesicles from proximal and distal small intestine. In the hamster, our previous in vivo studies had shown (1) both mediated and nonmediated components of calcium transport, (2) greater nonmediated transport in proximal than distal small intestines, and (3) $V_{\max }$ for calcium transport twice as great in distal as in proximal small intestine. In the present study with brush border membrane vesicles from hamster, $V_{\max }$ for saturable calcium transport was again twice as great in distal as in proximal small intestine. However, nonsaturable calcium transport rates relative to saturable rates were much greater with vesicles than in in vivo studies, and were greater in vesicles from distal than proximal small intestine. Since rates of saturable calcium uptake by brush border membrane vesicles parallel corresponding in vivo mediated transport rates, we conclude that the segmental rates of calcium transport in rat and hamster could be determined by brush border function.
\end{abstract}

\section{Introduction}

In most animals studied, absorption of calcium is more rapid at proximal than at distal sites in the small intestine. This is true for the rat (1-7), rabbit (2), guinea pig (2), mouse (2), and man (8). The hamster appears to be unique in showing a greater absorption rate in distal than in proximal small intestine (9).

We measured transcellular calcium transport in vivo in the steady state in rat (10) and hamster (9), and determined transport kinetics. In the rat we found $V_{\max }$ for mediated calcium transport to be more than twice as great in proximal as in distal small intestine. In the hamster we found $V_{\max }$ for mediated calcium transport to be twice as great in distal as in proximal small intestine. These prior studies measured transepithelial transport,

\footnotetext{
Address correspondence to Dr. Schedl. 1985.

Received for publication 23 October 1984 and in revised form 26 July
}

The Journal of Clinical Investigation, Inc.

Volume 76, November 1985, 1871-1878 which is the net result of movements of calcium across the apical and basal cell membranes, transit across the enterocyte, and paracellular diffusion. The cellular locus of the rate-limiting step that determines these differences between rat and hamster proximal and distal segments in carrier-mediated calcium transport actoss the cell is not known. We tested the possibility that the locus is the brush border by measuring kinetics of calcium uptake by brush border membrane vesicles prepared from proximal and distal small intestine of rat and hamster, and compared the findings with kinetics of transcellular calcium transport.

\section{Methods}

Animals. We used young, growing male animals. Rats and hamsters were obtained from Harlan-Sprague-Dawley. Animals were fed Purina chow 5012 (Ralston Purina Co., St. Louis, MO) (1.01\% calcium, $0.74 \%$ phosphorus, $0.20 \%$ magnesium, and $3.3 \mathrm{IU}$ vitamin $\mathrm{D}_{3} / \mathrm{g}$ ) ad lib. and weighed three times per week. Rats weighing $180-200 \mathrm{~g}$ and hamsters weighing $70-80 \mathrm{~g}$ were used for preparation of brush border membrane vesicles.

Preparation of vesicles. Animals had access to food up to the time of killing. They were weighed, killed by cervical dislocation, and the entire small intestine was removed. For rats, the proximal $30 \mathrm{~cm}$ and distal $20 \mathrm{~cm}$ were used to prepare vesicles. The most proximal and most distal 12-cm segments were used to prepare vesicles from the hamster. These segments were rinsed with ice-cold saline, everted, and placed on a glass plate resting on ice. The mucosa was scraped off with a glass microscope slide. 5 rats or 10 hamsters were usually used for a vesicle preparation.

Crude brush borders were isolated by the method of Forstner et al. (11). During the course of the preparation, brush border isolation was monitored by phase-contrast microscopy. To separate plasma membranes from adhering terminal web and microvillus core, we used the technique of Hopfer and collaborators (12), which allows purification of marker enzymes to two to three times greater specific activity than other methods. Briefly, the brush borders from $\sim 4 \mathrm{~g}$ of mucosa were suspended in 10 $\mathrm{ml}$ of $0.1 \mathrm{M}$ mannitol, $1 \mathrm{mM}$ Hepes-Tris, $\mathrm{pH}$ 7.4. Sodium thiocyanate was added from a concentrated solution $(4.5 \mathrm{M})$ to achieve a final concentration of $0.41 \mathrm{M}$. The suspension was mechanically homogenized at 2,000 rpm for 12 slow strokes in a glass-Teflon homogenizer. The suspension was diluted to a thiocyanate concentration of $50 \mathrm{mM}$ to reaggregate the cytoskeletal material, which was removed by centrifugation at $6,000 \mathrm{~g}$ for $10 \mathrm{~min}$. The membranes were then collected by centrifugation at $34,000 \mathrm{~g}$ for $25 \mathrm{~min}$. Residual sodium thiocyanate was washed out by resuspending the membranes in $0.1 \mathrm{M}$ mannitol, $1 \mathrm{mM}$ Hepes-Tris buffer, $\mathrm{pH}$ 7.4, or appropriate buffer used for uptake studies. The membranes were collected by centrifugation at $48,000 \mathrm{~g}$ for $20 \mathrm{~min}$. Protein concentration in this fraction was of the order of $3 \mathrm{mg} / \mathrm{ml}$.

Purification of brush border membrane vesicles was assessed by measurement of activities of sucrase (13) and alkaline phosphatase (14) expressed as units per gram protein. Alkàline phosphatase was measured in an EDTA-free homogenate of an aliquot of mucosa, and brush border membrane vesicles were diluted before assay in $0.1 \mathrm{mM} \mathrm{ZnCl}$ to replace zinc chelated in the original EDTA homogenate. Protein was measured by the method of Lowry et al. (15). Activity of $\mathrm{Na}^{+} \mathrm{K}^{+} \mathrm{ATPase}$ (16) was 
expressed as micromoles of inorganic phosphate (17) released per milligram protein per hour. $\mathrm{Na}^{+} \mathrm{K}^{+}$ATPase activity was measured as the difference between inorganic phosphate released in the presence and absence of ouabain. As an index of mitochondrial contamination, we measured cytochrome $c$ oxidase (18) and expressed the results as nanomoles cytochrome $c$ oxidized per minute per milligram protein.

Fo validate that the vesicles were closed and possessed the capability for transport, we measured sodium-coupled D-glucose uptake. ${ }^{14} \mathrm{C}$-Dglucose at a concentration of $1.62 \mathrm{mM}$ was present in the external medium only. Both media contained $1 \mathrm{mM}$ Hepes-Tris, and the internal medium contained $300 \mathrm{mM}$ mannitol in addition. The external medium was either $-\mathrm{Na}$, and contained $300 \mathrm{mM}$ mannitol, or was $+\mathrm{Na}$ and contained $165 \mathrm{mM}$ mannitol, $67 \mathrm{mM} \mathrm{NaSCN}$, and $6.7 \mathrm{mM} \mathrm{NaN}_{3}$.

Electron microscopy. Vesicles were fixed by addition of $2.5 \%$ glutaraldehyde in $0.1 \mathrm{M}$ sodium cacodylate buffer at $\mathrm{pH} 7.2$ to the suspension. After $30 \mathrm{~min}$ the vesicles were washed in $0.1 \mathrm{M}$ sodium cacodylate buffer at pH 7.2 (three changes) for $\sim 1 / 2 \mathrm{~h}$ in order to remove free aldehyde. The specimen was postfixed in $1 \%$ osmium tetroxide and $1.5 \%$ potassium ferrocyanide in $0.1 \mathrm{M}$ sodium cacodylate buffer at $\mathrm{pH} 7.2$ for $1 \mathrm{~h}$, and washed with $0.1 \mathrm{M}$ buffer followed by distilled water to remove free osmium. The specimen was stained en bloc with $2.5 \%$ uranyl acetate in distilled water for $5 \mathrm{~min}$, gradually dehydrated in acetone, embedded in Spurr's plastic, and polymerized at $70^{\circ} \mathrm{C}$ for $8 \mathrm{~h}$. The blocks were trimmed and thin sections were cut on a Reichert Ultracut $E$ ultramicrotome equipped with a DuPont diamond knife. Gray to light gold sections were mounted on 300 -mesh grids. The sections were stained with $5 \%$ uranyl acetate in water for 8-10 min, and secondarily stained with lead citrate for 6-8 $\mathrm{min}$. Vesicles were then examined and photographed in an Hitachi $\mathrm{H}-600$ microscope at $50 \mathrm{kV}$.

Transport studies. Calicum uptake: Calcium uptake was determined by a Millipore filtration technique. The composition of the incubation medium for calcium transport studies was $100 \mathrm{mM} \mathrm{KCl}, 5 \mathrm{mM} \mathrm{MgCl}$, and $20 \mathrm{mM}$ Hepes-Tris, $\mathrm{pH} 7.5$, with concentrations of calcium and ${ }^{45} \mathrm{Ca}$ as shown in captions to the figures. The stop solution was identical except for the addition of EGTA at a $1 \mathrm{mM}$ concentration. Usually 25 $\mu \mathrm{l}$ of membrane suspension was added to $125 \mu \mathrm{l}$ of incubation medium kept in a heater block at $25^{\circ} \mathrm{C}$. At serial time intervals, a $25-\mu$ l sample was removed from the incubation medium and diluted into $1 \mathrm{ml}$ of icecold stop solution. The diluted samples were immediately filtered through Millipore filters (type HA, $0.45 \mu \mathrm{m}$, diameter $25 \mathrm{~mm}$ ) and the filter was washed with $6 \mathrm{ml}$ of ice-cold stop solution. Radioactivity remaining on the filter was counted by liquid scintillation after dissolving the filter in $2 \mathrm{ml}$ of ethylene glycol monoethyl ether and adding $8 \mathrm{ml}$ of ACS (Amersham Corp., Arlington Heights, IL) scintillation solution. Calcium uptake was expressed as nanomoles of calcium per milligram of membrane protein per minute. Uptake rate at $12 \mathrm{~s}$ was used for all kinetic studies. Kinetic data were derived from four separate experiments using 5-10 animals per experiment.

Zero time points were determined by withdrawing a $25-\mu \mathrm{l}$ aliquot from the incubation medium and adding it to the stop solution, after which $25 \mu \mathrm{l}$ of membrane suspension was added. The mixture was filtered and washed and the filters were counted for radioactivity as described above. Zero-time point radioactivity was $0.03-0.05 \%$ of total radioactivity at $0.03 \mathrm{mM}$ calcium. Protein concentration of brush border membrane in the uptake medium was $\sim 500 \mu \mathrm{g} / \mathrm{ml}$. To determine intravesicular volume, the uptake of $1.62 \mathrm{mM}$ D-glucose was measured at $0.5,1.0$, and $2 \mathrm{~h}$ using the medium employed for calcium uptake studies. At 2 $\mathrm{h}$, glucose uptake had reached equilibrium, i.e., was the same as at $1 \mathrm{~h}$. Since we used a relatively high glucose concentration, glucose uptake due to binding was minimal, and it was assumed that the glucose was in an intravesicular compartment of the same concentration as in the medium. Intravesicular volume was calculated as follows: IV = (glucose uptake)/(medium glucose conc), where IV = intravesicular volume, microliter per milligram protein; glucose uptake $=$ nanomole per milligram protein at equilibrium $(1 \mathrm{~h})$; medium glucose concentration (conc) $=$ nanomoles per microliter.

We calculated kinetics of saturable and nonsaturable calcium transport as previously described (9). Total uptake rates were plotted against medium calcium concentration. These curves suggested that calcium uptake comprised saturable and nonsaturable components. The rate constant for nonsaturable calcium uptake, $K_{d}$ (nanomoles per hour per milligram protein), was calculated by the method of Neame and Richards (19). $K_{d}$ is assumed to be equivalent to the slope of the uptake curve at high medium concentrations, where total transport rates increase linearly with medium calcium concentrations. In these studies, rates increased linearly above $0.3 \mathrm{mM}$ and fit a straight line. The correlation coefficient was $r=0.9529(P<0.05)$ or better. Hence, the slope was determined by linear regression and taken as the diffusion component, $K_{d}$.

The saturable component of uptake was obtained by subtracting nonsaturable uptake at each calcium concentration $\left(K_{d} \times\right.$ calcium concentration) from total uptake. Saturable uptake was evaluated by the Lineweaver-Burk (20) and Hofstee (21) methods and was found to follow Michaelis-Menten kinetics. Hence, $K_{\mathrm{t}}$ (medium calcium concentration at half-maximal saturable calcium uptake rate) and $V_{\max }$ (the maximal rate of saturable calcium uptake at infinite medium calcium concentration) values were calculated.

Calcium efflux. The effect of ionophore A23187 on calcium efflux was measured using brush border membrane vesicles isolated in 100 $\mathrm{mM} \mathrm{KCl}, 5 \mathrm{mM} \mathrm{MgCl}$, and $1 \mathrm{mM}$ Hepes-Tris. Vesicles were incubated in the same medium containing $0.1 \mathrm{mM} \mathrm{Ca}^{++}$and ${ }^{45} \mathrm{Ca}$ for $5 \mathrm{~min}$ at $25^{\circ} \mathrm{C}$. To measure initial concentration ( $100 \%$ value at zero time), samples were removed into stop solution for filtration as in uptake studies. Either EGTA or A23187 plus EGTA was added at zero time to equal aliquots of ${ }^{45} \mathrm{Ca}$-loaded vesicles to give final concentrations of $0.5 \mathrm{mM}$ EGTA or $0.5 \mathrm{mM}$ EGTA containing $1 \mu \mathrm{g} / \mathrm{ml} \mathrm{A23187}$ in $0.01 \%$ dimethylsulfoxide. Samples were removed at $0.5,1,2,3,4,60,90$, and $120 \mathrm{~min}$ and efflux was expressed as the percentage of calcium remaining compared with the $100 \%$ value at zero time. Data are expressed as representative values from a series of experiments or as mean \pm 1 SE. Statistical analysis was by $t$ test.

\section{Results}

The properties of the brush border membrane vesicles used in these studies are shown in Table I. Sucrase activity in the mucosal homogenate of the rat was one-third to one-fifth that of the hamster. Enrichment of sucrase activity in vesicle preparations was greater in rat than hamster: sucrase activity was enhanced 46-fold in proximal small intestine of the rat and 31-fold in proximal small intestine of hamster. Sucrase enrichment did not differ in proximal as compared with distal small intestine in the rat, but was lower in distal than proximal small intestine in hamster. Alkaline phosphatase activity in mucosal homogenate from proximal small intestine was four times greater in rat than hamster, but was the same in both species in the distal segment. Enrichment factors for alkaline phosphatase were lower than for sucrase and greater in proximal than distal small intestine.

Intravesicular space determined by glucose uptake at equilibrium was $1.26 \mu \mathrm{l} / \mathrm{mg}$ protein for rat and 2.18 for hamster, i.e., in the range reported by other investigators $(12,22-25)$. Our space measurements were made at equilibrium in the medium we used for calcium uptake studies $(100 \mathrm{mM} \mathrm{KCl}, 5 \mathrm{mM}$ $\mathrm{MgCl}_{2}$, and $20 \mathrm{mM}$ Hepes-Tris, $\mathrm{pH}$ 7.5) with $1.62 \mathrm{mM}$ D-glucose added instead of calcium.

Ouabain-inhibitable $\mathrm{Na}^{+} \mathrm{K}^{+}$ATPase of the rat ranged from 0.8 to 2.4 in the homogenate and was enriched 1.4-fold in the vesicles. Ouabain-inhibitable $\mathrm{Na}^{+} \mathrm{K}^{+}$ATPase activity in the hamster was 0.20 in the homogenate and decreased to virtually undetectable levels, 0.08 , in the vesicles. Activity of cytochrome $c$ oxidase in the homogenate was $0.029 \pm 0.009 \mathrm{nmol}$ oxidized/ $\mathrm{mg}$ protein per min and decreased to one-fifth this value in the vesicle fraction. 
Table I. Intravesicular Space and Sucrase and Alkaline Phosphatase Activities during Preparation of Brush Border Membrane Vesicles (BBMV) from Rat and Hamster

\begin{tabular}{|c|c|c|c|c|}
\hline & \multicolumn{2}{|l|}{ Rat } & \multicolumn{2}{|l|}{ Hamster } \\
\hline & Proximal & Distal & Proximal & Distal \\
\hline & $30 \mathrm{~cm}$ & $20 \mathrm{~cm}$ & $12 \mathrm{~cm}$ & $12 \mathrm{~cm}$ \\
\hline \multicolumn{5}{|l|}{ Sucrase $(U / g$ protein $)$} \\
\hline Homogenate & $43 \pm 2$ & $6.4 \pm 0.8$ & $131 \pm 13$ & $32 \pm 3$ \\
\hline BBMV & $1,970 \pm 150$ & $263 \pm 48$ & $3,700 \pm 170$ & $740 \pm 80$ \\
\hline Enrichment Factor & $46 \pm 3$ & $39 \pm 6$ & $31 \pm 4$ & $20 \pm 2$ \\
\hline \multicolumn{5}{|l|}{ Alkaline phosphatase ( $U / g$ protein $)$} \\
\hline Homogenate & $820 \pm 160$ & $44 \pm 8$ & $203 \pm 51$ & 45 \\
\hline BBMV & $13,800 \pm 1,200$ & 295 & $4,080 \pm 1,250$ & 460 \\
\hline Enrichment factor & $18 \pm 2$ & 7 & $19 \pm 2$ & 10 \\
\hline Intravesicular space ( $\mu l / m g$ protein) & $1.26 \pm 0.13$ & 1.36 & $2.18 \pm 0.05$ & $1.97 \pm 0.08$ \\
\hline
\end{tabular}

Characterization of vesicles by transmission electron microscopy showed mostly closed vesicles. The appearance was similar for preparations from rat and hamster and for preparations from proximal and distal small intestine. Electron-dense material was absent from the vesicle interior, consistent with removal of cytoskeletal material. These vesicles may offer specific advantages for calcium transport studies: $(a)$ since calcium precipitation was not used for vesicle preparation, contamination by exogenous calcium was minimized; $(b)$ the removal of cytoskeletal material decreased intravesicular calcium binding.

To characterize transport behavior of vesicle preparations, we determined sodium-coupled D-glucose uptake. In the rat, a sharp glucose overshoot was present in vesicles from proximal intestine, but the overshoot was much lower in the distal small intestine (Fig. 1). In hamster, vesicles from both sites showed the overshoot (Fig. 2), which was greater with both segments than with vesicles from the rat proximal segment (Fig. 1).

The time course of calcium uptake by brush border membrane vesicles from proximal and distal small intestine of the rat at three different calcium concentrations is shown in Fig. 3. Uptake was most rapid initially, and the curves flattened at later time points. Uptake was more rapid with vesicles from the proximal than the distal segment, and the difference was more marked at the lower concentrations. The time course of uptake of calcium at three concentrations by brush border membrane vesicles prepared from proximal and distal small intestine of the hamster is shown in Fig. 4. In contrast with the corresponding data from the rat (Fig. 3), uptake was more rapid by vesicles from distal than proximal small intestine.

The effect of temperature on time course of calcium uptake is shown in Figs. 5 and 6. With vesicles from proximal and distal small intestine of the rat as well as from the entire small intestine of hamster, uptake was more rapid at $25^{\circ} \mathrm{C}$ than at $0^{\circ} \mathrm{C}$. The effect of the calcium ionophore A23187 on efflux of calcium from vesicles preloaded with ${ }^{45} \mathrm{Ca}$ is shown in Figs. 7 and 8 . Either EGTA or EGTA plus A23187 was added to vesicles preloaded with calcium and rate of efflux was measured. Efflux was greater in the presence of ionophore with vesicles from both proximal and distal segments of rat and hamster.

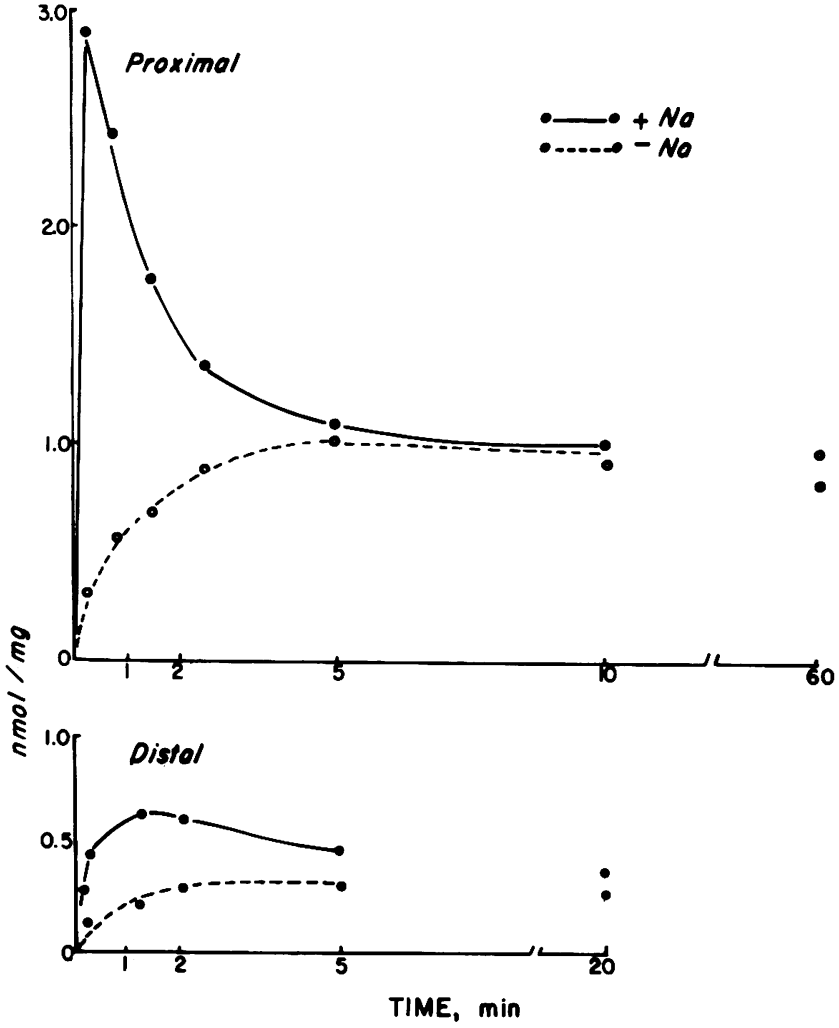

Figure 1. Rat data. Uptake of D-glucose nanomole per milligram protein by brush border membrane vesicles that were prepared from mucosa of the proximal $30 \mathrm{~cm}$ or distal $20 \mathrm{~cm}$ of small intestine. Internal and external vesicle media were isotonic. All media contained $1 \mathrm{mM}$ Hepes-Tris. Temperature: $25^{\circ} \mathrm{C}$. For all glucose studies, ${ }^{14} \mathrm{C}$-D-glucose at a concentration of $1.62 \mathrm{mM}$ was present in the external medium only. Internal medium was $300 \mathrm{mM}$ mannitol. External medium: - Na, $300 \mathrm{mM}$ mannitol; +Na, $165 \mathrm{mM}$ mannitol, $67 \mathrm{mM} \mathrm{NaSCN}$, and $6.7 \mathrm{mM} \mathrm{NaN}_{3}$. Vesicles from the proximal segment showed a well-defined sodium-coupled D-glucose overshoot followed by equilibration. The sodium-coupled D-glucose overshoot was much smaller with vesicles prepared from distal small intestine as compared with proximal. 


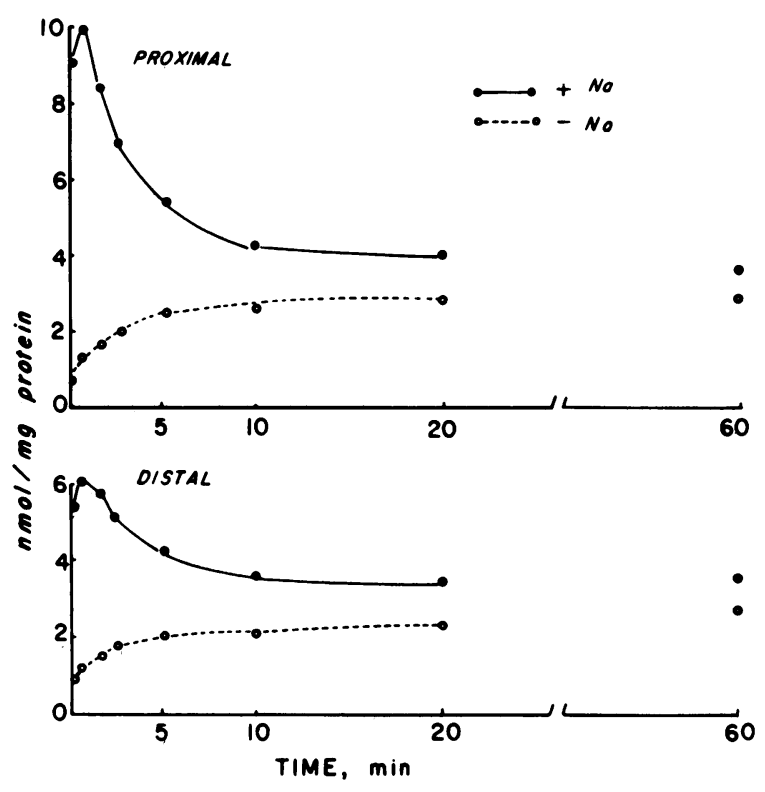

Figure 2. Hamster data. Uptake of D-glucose by brush border membrane vesicles prepared from the proximal or distal $12 \mathrm{~cm}$ of hamster intestine. Experimental conditions as described for rat above. Note greater overshoot with vesicles from hamster than with rat vesicles (Fig. 1). D-Glucose overshoot was greater with vesicles from the proximal than the distal small intestine.

Calcium uptake by vesicles from proximal and distal small intestine of the rat in relation to medium calcium concentration is shown in Fig. 9. Total uptake was slightly greater in proximal than in distal small intestine. When total transport was resolved
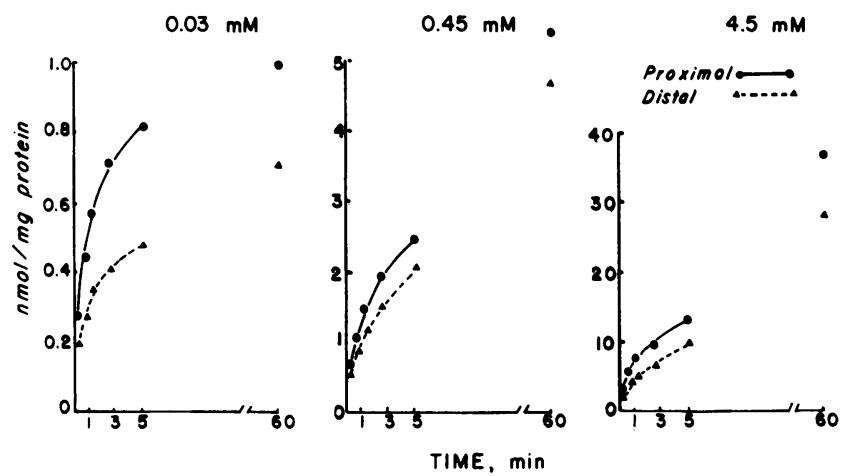

Figure 3. Rat data. Time course of calcium uptake by brush border membrane vesicles from mucosa of the proximal $30 \mathrm{~cm}$ and distal 20 $\mathrm{cm}$ of the small intestine. Vesicles contained $100 \mathrm{mM} \mathrm{KCl}, 5 \mathrm{mM}$ $\mathrm{MgCl}_{2}$, and $20 \mathrm{mM}$ Hepes-Tris, $\mathrm{pH}$ 7.5. The incubation medium was identical, except that calcium was added to give concentrations of $0.03,0.45$, and $4.5 \mathrm{mM}$. Temperature, $25^{\circ} \mathrm{C}$; time points, $0.2,0.75$, $1.5,2.5,5$, and $60 \mathrm{~min}$. Uptake was rapid during the first $5 \mathrm{~min}$ and continued at a slower rate during the subsequent $55 \mathrm{~min}$. Uptake by the proximal segment was much greater than by the distal segment, and the difference between segments was greater at the lower concentrations. Since uptake comprised saturable and nonsaturable components (see Fig. 9), and the saturable component made it greatest contribution to total transport at the lowest concentration, the curves at $0.03 \mathrm{mM} \mathrm{Ca}^{2+}$ demonstrated a major difference between the segments in saturable transport. This difference was obscured at the highest concentration, where most of the transport was nonsaturable, with mediated transport a minor component.

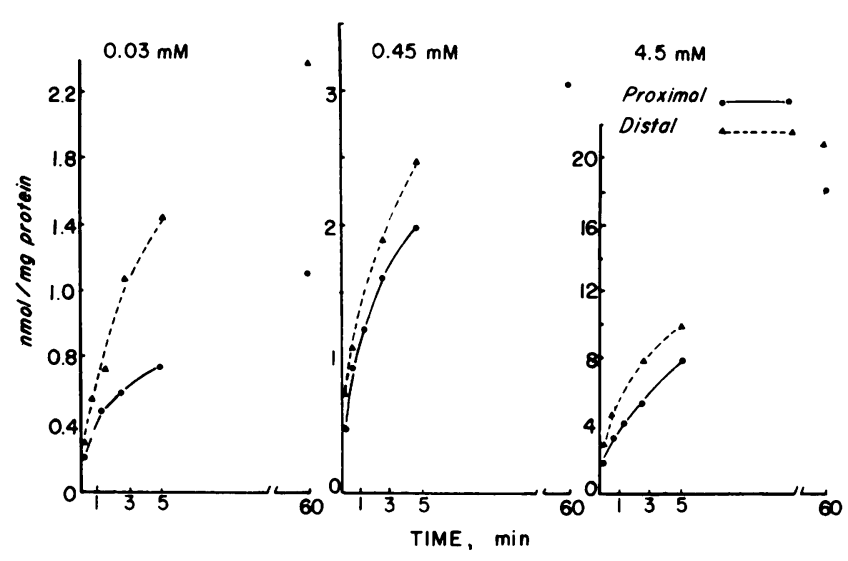

Figure 4. Hamster data. Time course of calcium uptake by brush border membrane vesicles from proximal and distal small intestine of the hamster. Conditions of the study are described in Fig. 3. Uptake was rapid during the first $5 \mathrm{~min}$ and continued at a reduced rate during the subsequent $55 \mathrm{~min}$. Uptake by the distal segment was much greater than by the proximal segment, and the difference between segments was greatest at the lowest concentration. Since uptake comprised saturable and nonsaturable components (Fig. 10), and the saturable component makes its greatest contribution to total uptake at the lowest concentration, the curves at $0.03 \mathrm{mM}$ calcium demonstrated a major difference between the segments in saturable transport. This difference between the segments was greatly decreased at $4.5 \mathrm{mM}$ calcium, where nonsaturable transport was the major component.

into saturable and nonsaturable components, saturable transport was much greater in proximal than distal small intestine, as shown by $V_{\max }$ data. Nonsaturable transport was the same in both segments (Table II).

Fig. 10 shows calcium uptake by membrane vesicles from proximal and distal small intestine of the hamster in relation to

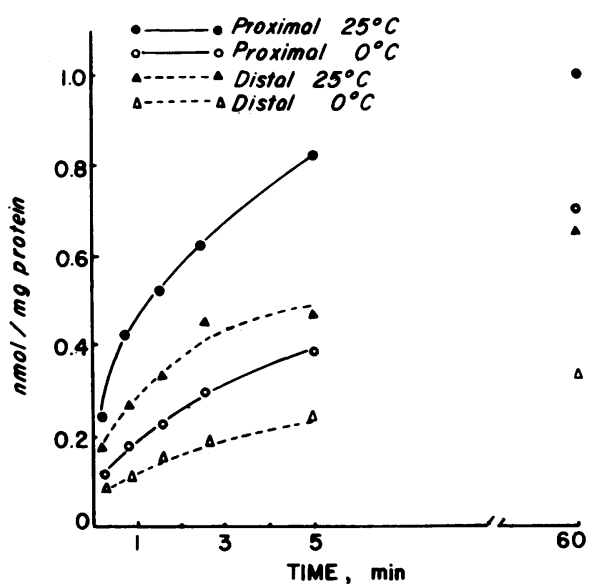

Figure 5. Rat data. Time course of $\mathrm{Ca}^{2+}$ uptake by brush border membrane vesicles from mucosa of proximal $30 \mathrm{~cm}$ and distal $20 \mathrm{~cm}$ of small intestine: effect of temperature. $\mathrm{Ca}^{2+}$ concentration was 0.030 $\mathrm{mM} . \mathrm{Ca}^{2+}$ uptake was markedly temperature dependent and was decreased to half the rate at $25^{\circ} \mathrm{C}$ by cooling to $0^{\circ} \mathrm{C}$. Since $\mathrm{Ca}^{2+}$ binding to the vesicle surface would be expected to increase at the lower temperature, $\mathrm{Ca}^{2+}$ uptake was not simple binding to the vesicle surface. Decreased $\mathrm{Ca}^{2+}$ uptake at the lower temperature was most consistent with an effect on transmembrane transport of $\mathrm{Ca}^{2+}$ caused by decreased membrane fluidity. 


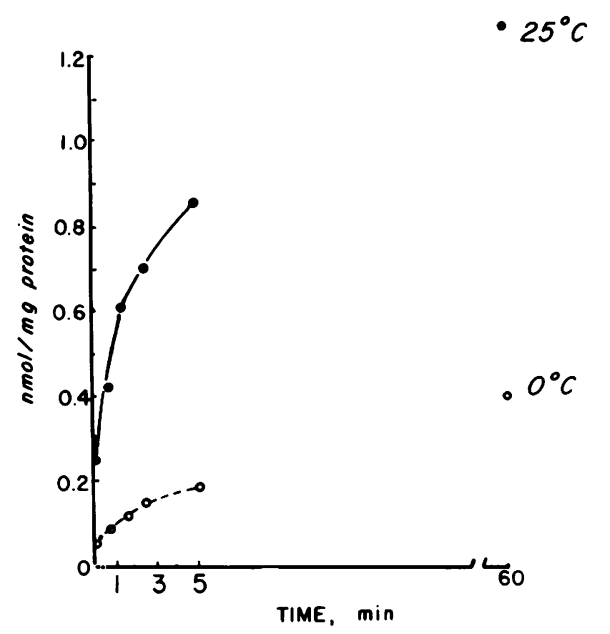

Figure 6. Hamster data. Time course of $\mathrm{Ca}^{2+}$ uptake by brush border membrane vesicles from entire small intestine of hamster: effect of temperature. Vesicles contained $100 \mathrm{mM} \mathrm{KCl}, 5 \mathrm{mM} \mathrm{MgCl}_{2}$, and 20 $\mathrm{mM}$ Hepes-Tris, $\mathrm{pH}$ 7.5. The incubation medium was identical except for $\mathrm{Ca}^{2+}$ concentration of $0.03 \mathrm{mM}$. Calcium uptake was markedly lower at $0^{\circ} \mathrm{C}$ than at $25^{\circ} \mathrm{C}$.

concentration. In contrast to the rat, total and saturable calcium uptake was much greater in vesicles from distal than from proximal small intestine. Mean rates of saturable calcium uptake were greater in vesicles from distal intestine of hamster than proximal intestine of rat. Similarly, at the sites of lower uptake rates, mean rates of saturable uptake of calcium by vesicles from proximal small intestine of hamster were greater than by vesicles from distal intestine of the rat.

\section{Discussion}

To establish calcium transport behavior of the small intestine, we previously measured steady state in vivo transcellular calcium
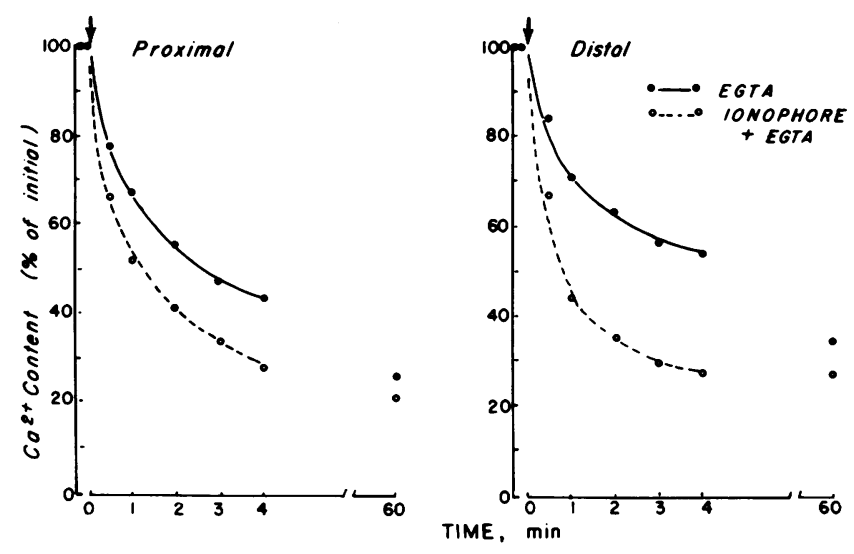

Figure 7. Rat data. Efflux of $\mathrm{Ca}^{++}$from brush border membrane vesicles from proximal and distal small intestine. Vesicles were incubated in $0.1 \mathrm{mM} \mathrm{CaCl}$ and ${ }^{45} \mathrm{Ca}$ for $5 \mathrm{~min}$ at $25^{\circ} \mathrm{C}$ in $100 \mathrm{mM} \mathrm{KCl}, 5 \mathrm{mM}$ $\mathrm{MgCl}_{2}$, and $1 \mathrm{mM}$ Tris-Hepes, $\mathrm{pH}$ 7.5. To obtain the $100 \%$ value at the zero time point, samples were removed before addition of either EGTA or calcium ionophore A23187. The arrow (.) indicates addition of EGTA or EGTA plus ionophore. Medium concentrations: EGTA in both media, $0.5 \mathrm{mM}$; EGTA and ionophore also contained A23187 $(1 \mu \mathrm{g} / \mathrm{ml})$ in $0.01 \%$ dimethylsulfoxide. Efflux was calculated as percentage of initial amount of ${ }^{45} \mathrm{Ca}$ remaining at $0.5,1.0,2.0,3.0,4.0$, and $60 \mathrm{~min}$. In this experiment, vesicle protein concentration was 244 $\mu \mathrm{g} / \mathrm{ml}$ in the proximal medium and $256 \mu \mathrm{g} / \mathrm{ml}$ in the distal.

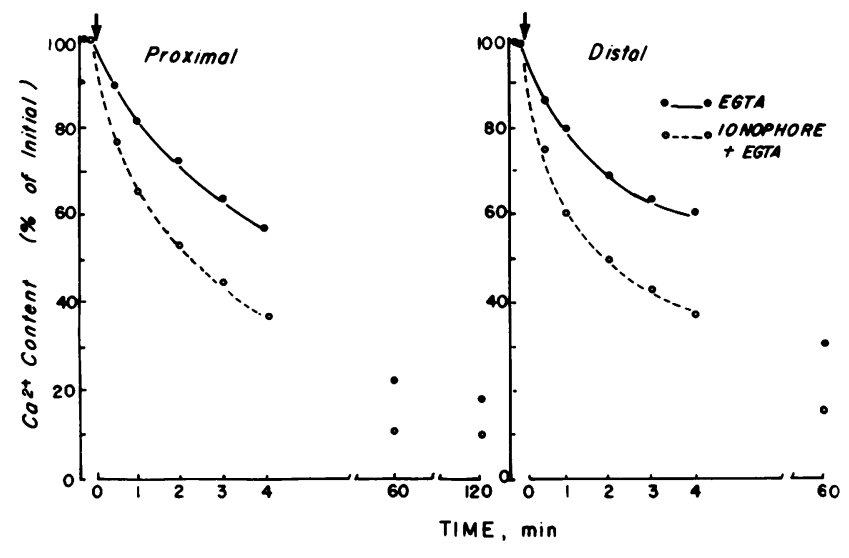

Figure 8. Hamster data. Efflux of $\mathrm{Ca}^{++}$from brush border membrane vesicles from proximal and distal small intestine. Experiments were performed as described in the legend for Fig. 7. Protein concentration of vesicles in proximal medium was $806 \mu \mathrm{g} / \mathrm{ml}$; in distal medium it was $123 \mu \mathrm{g} / \mathrm{ml}$.

transport in proximal and distal small intestine of rat and hamster. In those studies, we found $V_{\max }$ for calcium transport to be greater in proximal than in distal small intestine in the rat (10), and the reverse to be true in hamster (9) (Table III). To test whether luminal membrane function determined these differences in transport, we prepared brush border membrane vesicles from these segments in the two species and determined calcium uptake kinetics. As shown in Table II, $V_{\max }$ for saturable calcium uptake by vesicles parallels the $V_{\max }$ for transcellular calcium transport (Table III). Thus, influx at the brush border could be the rate-determining step in calcium transport in vivo.

Saturable calcium transport does not correlate with degree of purification of brush border membrane vesicles as assessed by enhancement of sucrase activity. Thus, $V_{\max }$ for saturable calcium uptake was greatest in vesicles from distal small intestine of hamster, the site with lowest enhancement of sucrase activity of vesicles. Therefore, it could be assumed that the difference between $V_{\max }$ values of vesicles from proximal and distal segments would be even greater if purification at both sites were equal. Sucrase activity enhancement is greater in vesicles from rat than hamster, but calcium transport activity is lower. The difference in enhancement of sucrase activity between vesicles from proximal and distal segments of rat is not significant and cannot explain the large difference in $V_{\max }$ values.

Transport behavior of membrane vesicles and intact enterocytes do not change in parallel with respect to nonsaturable transport. Calcium permeability or binding of calcium by vesicles differs from that of the intact enterocyte as measured by $K_{\mathrm{d}}$. For the rat, nonsaturable transport is not detectable by the in vivo steady state perfusion technique, because it is too small relative to mediated transport. However, nonsaturable transport is a major component of calcium uptake by brush border membrane vesicles (Table II, Fig. 9). For the hamster, in vivo nonsaturable transport is greater in duodenum than ileum (Table III), whereas the reverse is true for calcium transport by brush border membrane vesicles (Table II). Thus, preparation of vesicles changes membrane properties with respect to nonsaturable calcium transport. The greater permeability or binding of calcium by vesicles as compared with the corresponding intact segments in vivo is shown by data from the hamster. In vivo, the ratio of 


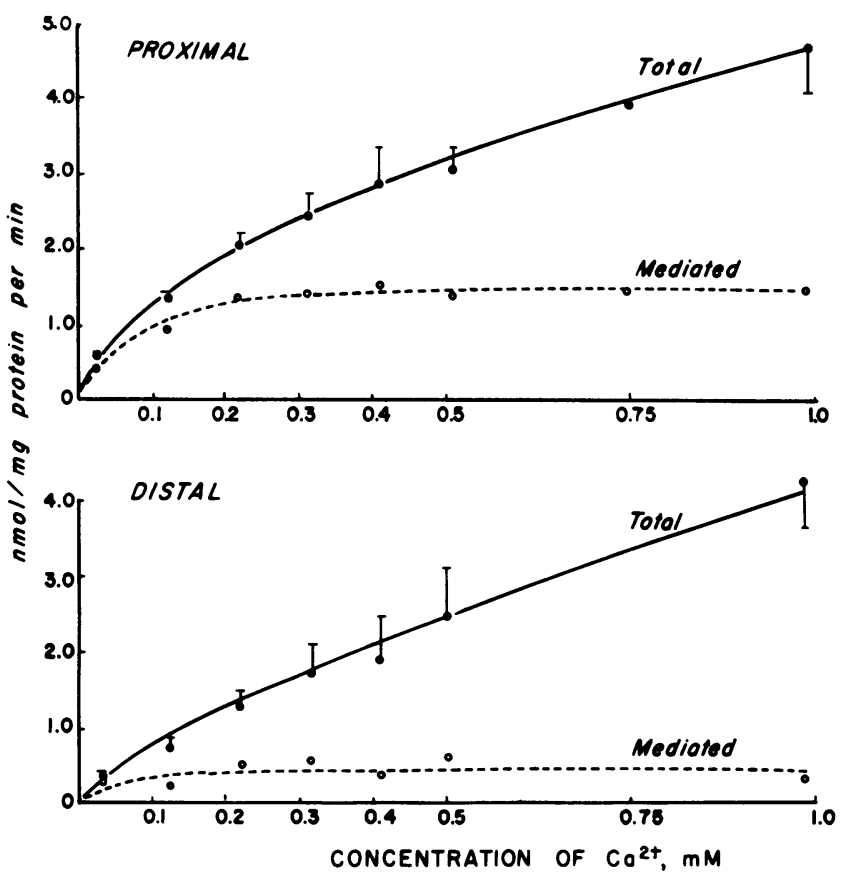

Figure 9. Rat data. Calcium uptake, nanomoles per milligram protein per minute, by brush border membrane vesicles from proximal $30 \mathrm{~cm}$ and distal $20 \mathrm{~cm}$ of the small intestine in relation to concentration. Incubation time was $12 \mathrm{~s}$. Mean data $( \pm \mathrm{SE})$ was from four experiments, each using 8-10 rats. The identical medium was present on both sides of the vesicles (legend, Fig. 3), except for calcium, which was present at the indicated concentrations in the external medium. Total calcium uptake continued to increase at the high external medium concentrations, which was consistent with a nonsaturable component of uptake. The slope of the line obtained by regressing the high concentration data points was used to calculate nonsaturable transport at each concentration. Nonsaturable transport data were subtracted from total transport data to obtain the data points for saturable transport. Since saturable transport conformed to Michaelis-Menten kinetics, $K_{\mathrm{t}}$ and $V_{\max }$ were calculated by the method of Lineweaver and Burk (20), and the curve for saturable transport was drawn using the kinetic constants (Table II). Calcium transport was predominantly saturable at low external medium concentrations, but the nonsaturable component predominated at high concentrations in the proximal segment. In the distal segment, saturable calcium uptake at $V_{\max }$ was onefourth that of the proximal segment, but the slope for nonsaturable transport was the same in both segments.

nonsaturable to saturable calcium transport is 0.25 in the proximal and 0.10 in the distal segment at $1.0 \mathrm{mM}$ calcium. At the same calcium concentration, with membrane vesicles, the ratio is 0.70 in proximal and 1.4 in distal. It should be noted that the greater permeability or calcium binding with vesicles occurs in the absence of the paracellular pathway and voltage gradient across the membrane present with in vivo studies.

The method of preparation of brush border membrane vesicles used in the current study differed from that employed in prior studies of the small intestine. Although Miller and Bronner (22) used a similar method, they did not remove cytoskeletal material as we did by the method of Hopfer et al. (12). They prepared duodenal vesicles from male rats fed a low calcium $(0.06 \%)$ and low phosphorus $(0.2 \%)$ semisynthetic diet for several days. Miller and Bronner (22) found that calcium uptake at 0.5 min showed saturation kinetics. Scatchard-plot analysis was used to define high $\left(K_{\mathrm{a}} 2.7 \times 10^{4} \mathrm{M}^{-1}\right)$ and low $\left(K_{\mathrm{a}} 60 \mathrm{M}^{-1}\right)$ affinity
Table II. Kinetic Constants for Calcium Uptake by Brush Border Membrane Vesicles from Proximal and Distal Small Intestine of Rat and Hamster (Mean $\pm S E$ )

\begin{tabular}{llll}
\hline Segment & $K_{\mathrm{t}}$ & $V_{\text {mex }}$ & $K_{\mathrm{d}}$ \\
\hline & $m M$ & $\begin{array}{l}\text { nmol/mg } \\
\text { protein } / \mathrm{min}\end{array}$ & $\begin{array}{l}\text { nmol/mg protein } \\
\mathrm{min} / \mathrm{mmol}\end{array}$ \\
Rat & & $1.62 \pm 0.06$ & 3.37 \\
$\quad \begin{array}{l}\text { Proximal } \\
\text { Distal }\end{array}$ & $0.067 \pm 0.014$ & $0.45 \pm 0.11^{*}$ & 3.65 \\
Hamster & $0.036 \pm 0.057$ & & \\
$\quad \begin{array}{l}\text { Proximal } \\
\text { Distal }\end{array}$ & $0.076 \pm 0.026$ & $1.15 \pm 0.08 \ddagger$ & 0.90 \\
& $0.075 \pm 0.017$ & $2.34 \pm 0.11 \ddagger$ & 3.05 \\
\hline
\end{tabular}

Concentration range $0.029-1 \mathrm{mM}$ calcium.

* Differs from the proximal segment, $P<0.05$ or less.

$¥$ Differs from the corresponding segment in the rat, $P<0.05$ or less.

binding sites. Rasmussen et al. (26) prepared duodenal vesicles from cockerels raised on a vitamin D-deficient diet in a darkened room for $4 \mathrm{wk}$. The cockerels were vitamin $\mathrm{D}$ repleted by administration of $1 \alpha$-hydroxycholecalciferol. Enterocytes were disrupted by homogenization in hypotonic buffer. Both sucrose gradient and glycerol gradient centrifugation were used for preparing purified membrane fragments. Rasmussen et al. (26) examined time course of calcium accumulation as a function of calcium concentration and found very little calcium accumulation below $0.2 \mathrm{mM}$ calcium. Rate of calcium accumulation was determined by subtracting 1-min uptake values from those

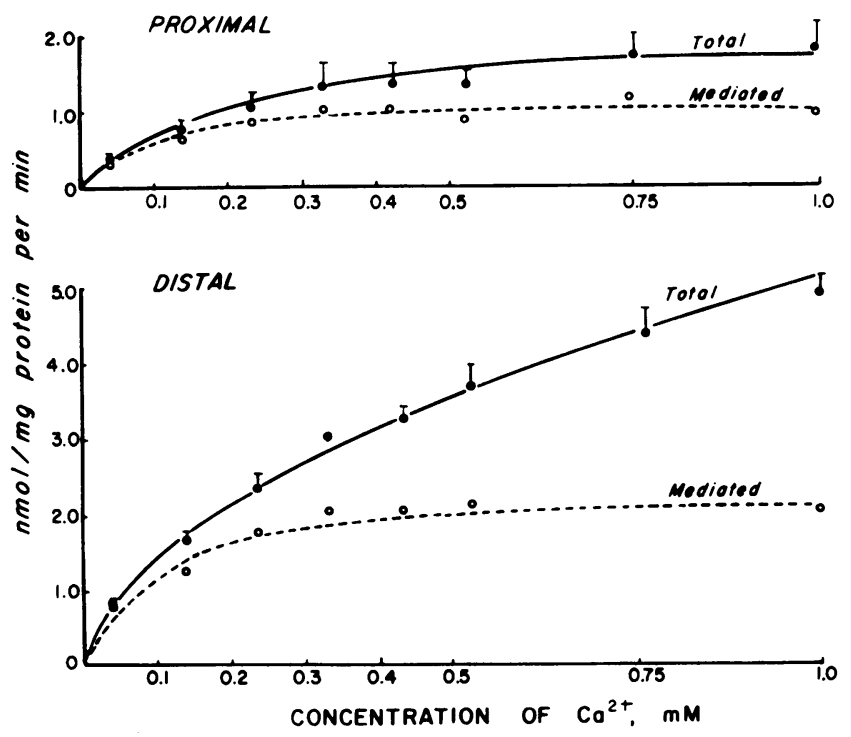

Figure 10. Hamster data. Calcium uptake by brush border membrane vesicles from the most proximal and most distal $12 \mathrm{~cm}$ of hamster intestine. Mean data ( \pm SE) was from four experiments, each using 8-10 hamsters. Conditions and method for calculating data is as described in Fig. 9. Saturable transport data points conformed closely to the curves calculated from the kinetic constants in both segments. Saturable calcium transport was greater in the distal than the proximal segment, which is the reverse of the situation in the rat (Fig. 9). Nonsaturable calcium transport was greater in the distal than the proximal segment, unlike the rat, where both segments showed the same $K_{d}$. 
Table III. Kinetic Constants for In Vivo Steady State Transcellular Calcium Transport by Proximal and Distal Small Intestine of Rat and Hamster (Mean Data)

\begin{tabular}{|c|c|c|c|}
\hline Segment & $K_{\mathrm{t}}$ & $V_{\max }$ & $K_{\mathrm{d}}^{*}$ \\
\hline & $m M$ & $\begin{array}{l}\mu \mathrm{mol} / \mathrm{g} d r y \mathrm{wt} \\
\text { segment/h}\end{array}$ & $\begin{array}{l}\mu \mathrm{mol} / \mathrm{g} d r y \text { wt } \\
\text { segment } / \mathrm{h} / \mathrm{mmol}\end{array}$ \\
\hline \multicolumn{4}{|l|}{ Rat } \\
\hline Proximal & $0.47 \pm 0.10$ & $38 \pm 2$ & \\
\hline Distal & $0.27 \pm 0.04 \ddagger$ & $15 \pm 0.5 \ddagger$ & \\
\hline \multicolumn{4}{|l|}{ Hamster } \\
\hline Proximal & $4.3 \pm 1.4$ & $137 \pm 16$ & 7.3 \\
\hline Distal & $4.3 \pm 1.3$ & $290 \pm 32 \ddagger$ & 2.9 \\
\hline
\end{tabular}

Concentration range $0-6.8 \mathrm{mM}$ for rat (10); 0-27 $\mathrm{mM}$ for hamster (9).

* Nonmediated component of calcium transport not present in rat in vivo.

$\ddagger$ Proximal and distal segments differ significantly, $P<0.05$ or less.

at later time points. Accumulation at 5 and $10 \mathrm{~min}$ was used to determine initial rate of calcium uptake. By this method of analysis, $K_{\mathrm{t}}$ was $1.4 \mathrm{mM}$ and $V_{\max }$ was $0.65 \mathrm{nmol} / \mathrm{mg}$ protein per min for vitamin D-replete animals. The investigators concluded that these values were approximate because of the nature of the system. Bikle et al. (27) fed cockerels a vitamin D-deficient diet containing $1 \%$ calcium and $0.45 \%$ phosphorus for $6 \mathrm{wk}$. To study the D-repleted state, cockerels received $1,25-(\mathrm{OH})_{2} \mathrm{D}_{3} 12 \mathrm{~h}$ before study. Duodenal brush border membrane vesicles were prepared by the method of Rasmussen et al. (26). Bikle et al. (27) used the Eadie-Hofstee plot to analyze rate of calcium accumulation as a function of calcium concentration and found a nonlinear relationship. As the measure of calcium uptake, they used calcium accumulation during the interval from 1 to $5 \mathrm{~min}$, which showed a nearly linear relationship with time. At submillimolar calcium concentrations, the $K_{\mathrm{t}}$ for calcium accumulation was $1.1 \times 10^{-4} \mathrm{M}$ for vesicles from 1,25-(OH) $)_{2} \mathrm{D}_{3}$-treated chicks.

Our studies cannot be compared directly with prior work. Miller and Bronner (22) studied vesicles from rats stimulated by low calcium and low phosphorous diet, rather than from animals maintained under usual laboratory conditions. Rasmussen (26) and Bikle (27) studied the chick, which appears to behave differently from the rat. Vesicles from prior studies showed lesser enhancement of marker enzymes, and calcium uptake may have been underestimated due to long incubation times.

Cell membranes are characterized by high affinity and capacity for binding calcium. Thus, uptake of calcium by brush border vesicles will be determined in part by extent and locus (intravesicular or extravesicular) of binding as well as transmembrane transport. Two types of experiments examined transmembrane transport of calcium in relation to binding: (1) temperature dependence of uptake; and (2) the effect of the calcium ionophore A23187 on efflux of calcium from preloaded vesicles. Since membrane transport of calcium depends in part on membrane fluidity (28), lowering the temperature and decreasing membrane fluidity should decrease transmembrane calcium transport. The decrease in calcium uptake at $0^{\circ} \mathrm{C}$ as compared with $25^{\circ} \mathrm{C}$ (Figs. 5 and 6) is consistent with the concept that a major component of calcium uptake is movement across the vesicle membrane. Further evidence for transmembrane movement of calcium during the uptake process is provided by experiments with the calcium ionophore A23187. Calcium efflux from preloaded vesicles was markedly enhanced by the calcium channels provided by the ionophore (Figs. 7 and 8 ).

We characterized the vesicles used in these studies with respect to marker enzymes (Table I) and sodium driven D-glucose uptake (Figs. 1 and 2). In addition to sucrase and alkaline phosphatase activity, we also measured $\mathrm{Na}^{+} \mathrm{K}^{+} \mathrm{ATPase}$. Sucrase enrichment (Table I) is several times greater with the Hopfer procedure (12) than the calcium or magnesium precipitation methods. Ouabain-inhibitable $\mathrm{Na}^{+} \mathrm{K}^{+}$ATPase activity was relatively low (29), and a low enrichment factor (we found 1.4 for rat) has been observed by other investigators (30). In hamster, $\mathrm{Na}^{+} \mathrm{K}^{+}$ATPase activity in brush border membrane vesicles declined below the level in homogenate.

Each of the four segments we studied yielded vesicles that showed sodium-driven D-glucose overshoot (Figs. 1 and 2). Vesicles from the distal segment of rat intestine showed a relatively small overshoot in comparison with rat proximal and hamster proximal and distal intestine. To estimate intravesicular volume present during our calcium studies, we measured intravesicular space using D-glucose under the conditions of our calcium uptake studies, i.e., no gradients except for calcium concentration (Table I). The larger intravesicular space in the hamster would tend to increase calcium uptake by maintaining the transvesicular calcium gradient longer than vesicles from the rat, and would accommodate more calcium at equilibrium.

We studied calcium uptake at the time points $0.2,0.75,1.5$, $2.5,5$, and $60 \mathrm{~min}$ to characterize the time course of calcium uptake. These studies showed greater uptake by vesicles from proximal than from distal segments in rat (Fig. 3) and the reverse for hamster (Fig. 4) at all time points. For kinetic studies, 0.2min uptake periods were used. At this time point, appreciable uptake of radioactivity had occurred, even at the higher calcium concentrations. Even at $0.2 \mathrm{~min}$, much of the calcium uptake must represent bound calcium, rather than calcium in solution within the vesicle. At the lowest medium concentration studied, $0.03 \mathrm{mM}$, assuming that the calcium taken up was dissolved in the mean intravesicular volume, for vesicles from rat proximal and hamster distal intestine, intravesicular calcium concentration is fourteen times that in the medium. In rat distal and hamster proximal small intestine, intravesicular calcium concentration is seven times that in the medium. At the highest medium calcium concentration studied, $1 \mathrm{mM}$, intravesicular calcium concentration would be four and three times medium concentration in rat proximal and distal vesicles, respectively, and equal to medium in hamster proximal and twice medium in hamster distal. At later time points, these calculated concentrations are considerably higher.

These studies comparing transepithelial and vesicular calcium transport show parallelism between saturable calcium transport in two differing species at two intestinal sites. They suggest that the transepithelial rate is determined by the calcium load admitted to the cell by the brush border. Further studies are required to validate these findings, since vesicles do not mimic in vivo transport with regard to nonsaturable transport: calcium permeability or binding of calcium to brush border membrane vesicles $(a)$ is greater than that of the corresponding intact segments in vivo, and $(b)$ does not show the same relationships among segments that are found in vivo. 


\section{Acknowledgments}

We thank Dr. Robert Crane, Dr. Ulrich Hopfer, and Dr. Rolf Kinne and their staff for advice and assistance in preparation of brush border membrane vesicles, and the Central Electron Microscopy Facility at the University of Iowa. Drs. Victor Schuster and Russel Husted reviewed the manuscript. Ionophore A23187 was supplied by the Eli Lilly \& Co., Indianapolis, IN.

This work was supported in part by the Research Service of the Veterans Administration, and by grant AM34986. T. Miller was a student fellow at the University of Iowa College of Medicine.

\section{References}

1. Schachter, D., and S. M. Rosen. 1959. Active transport of $\mathrm{Ca}^{45}$ by the small intestine and its dependence on vitamin D. Am. J. Physiol. 196:357-362.

2. Schachter, D., E. B. Dowdle, and H. Schenker. 1960. Active transport of calcium by the small intestine of the rat. Am. J. Physiol. 198: 263-268.

3. Krawitt, E. L., and H. P. Schedl. 1968. In vivo calcium transport by rat small intestine. Am. J. Physiol. 214:232-236.

4. Younoszai, M. K., and H. P. Schedl. 1972. Intestinal calcium transport: comparison of duodenum and ileum in vivo in the rat. Gastroenterology. 62:565-571.

5. Petith, M. M., and H. P. Schedl. 1976. Duodenal and ileal adaptation to dietary calcium restriction: in vivo studies in the rat. Am. J. Physiol. 231:865-871.

6. Petith, M. M., J. R. Wenger, and H. P. Schedl. 1978. Vitamin D dependence and aboral gradient of in vivo intestinal calcium transport in the rat. Am. J. Dig. Dis. 23:943-947.

7. Walker, B., and H. P. Schedl. 1979. Small intestinal calcium absorption in the rat with experimental diabetes. Proc. Soc. Exp. Biol. Med. 161:144-152.

8. Vergne-Marini, P., T. F. Parker, C. Y. C. Pak, A. R. Hull, H. F. DeLuca, and J. S. Fordtran. 1976. Jejunal and ileal calcium absorption in patients with chronic renal disease. J. Clin. Invest. 57:861-866.

9. Iskandarani, M., D. L. Miller, and H. P. Schedl. 1981. Kinetics of calcium transport in the hamster duodenum and ileum. Gastroenterology. 81:903-909.

10. Schedl, H. P., D. L. Miller, J. M. Pape, R. L. Horst, and H. D. Wilson. 1984. Calcium and sodium transport and vitamin D metabolism in the spontaneously hypertensive rat. J. Clin. Invest. 73:980-986.

11. Forstner, G. G., S. M. Sabesin, and K. J. Isselbacher. 1968. Rat intestinal microvillus membranes. Purification and biochemical characterization. Biochem. J. 106:381-390.

12. Hopfer, U., T. D. Crowe, and B. Tandler. 1983. Purification of brush border membrane by thiocyanate treatment. Anal. Biochem. 131: 447-452.

13. Younoszai, M. K., and H. P. Schedl. 1972. Effects of diabetes on intestinal disaccharidase activity. J. Lab. Clin. Med. 79:579-586.
14. Bowers, G. N., and R. B. McComb. 1966. A continuous spectrophotometric method of measuring the activity of serum alkaline phosphatase. Clin. Chem. 12:70-89.

15. Lowry, O. H., N. J. Rosebrough, A. L. Farr, and R. J. Randall. 1951. Protein measurement with the Folin phenol reagent. J. Biol. Chem. 193:265-275.

16. Guernsey, D. L., C. Boric, and I. S. Edelman. 1981. Crucial role of thyroid hormone in X-ray induced neoplastic transformation in cell culture. Proc. Natl. Acad. Sci. USA. 78:5708-5711.

17. Fiske, C. H., and Y. Subbarow. 1925. The colorimetric determination of phosphorus. J. Biol. Chem. 66:375-400.

18. Wharton, D. C., and A. Tzagoloff. 1967. Cytochrome $c$ oxidase (EC 1.9.3.1) Cytochrome C: $\mathrm{O}_{2}$ oxidoreductase. Methods Enzymol. 10: 245-250.

19. Neame, K. D., and T. G. Richards. 1972. Elementary Kinetics of Membrane Carrier Transport. Blackwell Scientific Publications, Oxford, England. 41, 52.

20. Lineweaver, H., and D. Burk. 1934. The determination of enzyme dissociation constants. J. Am. Chem. Soc. 56:658-666.

21. Hofstee, B. H. J. 1959. Non-inverted versus inverted plots in enzyme kinetics. Nature (Lond.). 184:1296-1298.

22. Miller, A., and F. Bronner. 1981. Calcium uptake in isolated brush-border vesicles from rat small intestine. Biochem. J. 196:391-401.

23. Murer, H., and U. Hopfer. 1974. Demonstration of electrogenic $\mathrm{Na}^{+}$-dependent D-glucose transport in intestinal brush border membranes. Proc. Natl. Acad. Sci. USA. 71:484-488.

24. Murer, H., U. Hopfer, E. Kinne-Saffran, and R. Kinne. 1974. Glucose transport in isolated brush-border and lateral-basal plasmamembrane vesicles from intestinal epithelial cells. Biochim. Biophys. Acta. 345:170-179.

25. Hopfer, U., K. Nelson, J. Perrotto, and K. Isselbacher. 1973. Glucose transport in isolated brush border membrane from rat small intestine. J. Biol. Chem. 248:25-32.

26. Rasmussen, H., O. Fontaine, E. Max, and D. B. P. Goodman. 1979. The effect of 1-hydroxyvitamin $D_{3}$ administration on calcium transport in chick intestine brush border membrane vesicles. J. Biol. Chem. 254:2993-2999.

27. Bikle, D. D., S. Munson, and D. T. Zolock. 1983. Calcium flux across chick duodenal brush border membrane vesicles: regulation by 1,25-dihydroxyvitamin D. Endocrinology. 113:2072-2080.

28. Holmes, R. P., M. Mahfouz, B. D. Travis, N. L. Yoss, and M. J. Keenan. 1983. The effect of membrane lipid composition on the permeability of membranes to $\mathrm{Ca}^{2+}$. Ann. NY Acad. Sci. 414:44-56.

29. Mircheff, A. K., and E. Wright. 1976. Analytical isolation of plasma membranes of intestinal epithelial cells: identification of $\mathrm{Na}, \mathrm{K}$ ATPase rich membranes and the distribution of enzyme activities. $J$. Membr. Biol. 28:309-333.

30. Maenz, D. D., and G. W. Forsyth. 1982. Ricinoleate and deoxycholate are calcium ionophores in jejunal brush border vesicles. J. Membr. Biol. 70:125-133. 